\title{
Surgical Treatment of Insertional Achilles Tendinosis and Haglunds Deformity by Using Central Tendon-splitting Approach: Retrospective Case Series of 15 Cases
}

\author{
Malhar Dave ${ }^{1}$, Heloni M Dave ${ }^{2}$, Rakesh Rathava ${ }^{3}$, Niravkumar P Moradiya ${ }^{4}$
}

\begin{abstract}
Purpose of the study: Haglund's deformity or pump bump is a degenerative process and is a common cause of posterior heel pain. Operative treatment is required when conservative treatment fails and the symptoms are not relieved. Different surgical procedures and approaches have been used for this deformity. But in the literature, the results have been inconsistent. In this study, we retrospectively evaluated the clinical and functional outcome of operative treatment with debridement of the retrocalcaneal bursa and the Achilles tendon using a central tendon-splitting approach.

Materials and methods: A total of 15 patients that underwent a surgical procedure were included in this study. The study duration was from January 2006 to June 2011. The clinical and functional outcome was evaluated using the American Orthopedics Foot and Ankle Society (AOFAS) score. All patients were operated in the prone position by using a central tendon-splitting approach.

Results: The mean follow-up period was 26 months. The mean AOFAS ankle-hind foot score had improved by 33 points from the preoperative mean score (37), with a mean score of 70 at the final follow-up. No complications (wound dehiscence and tendon avulsion) were noted. Out of 15 patients, all patients had good results except one due to persistent pain.

Conclusion: We concluded that the central approach to surgical correction is an effective method with a good clinical and functional outcome in patients with refractory Haglund's deformity.

Keywords: Achilles tendinosis, Chronic achilles tendinopathy, Haglund's deformity.

Journal of Foot and Ankle Surgery (Asia-Pacific) (2019): 10.5005/jp-journals-10040-1099
\end{abstract}

\section{INTRODUCTION}

Haglund's deformity which is also known as Mulholland deformity or pump bump or retrocalcaneal exostosis was first described by Patrick Haglund in the year 1927. It is a degenerative process characterized by osteophyte formation, retrocalcaneal bursitis, and calcifying metaplasia ${ }^{10,11}$ without evidence of inflammation at the tendon insertion site. The anatomical location of the retrocalcaneal bursa is between posterior and superior aspects of the tuberosity of the calcaneus and the anterior aspect of the distal Achilles tendon.

It is a common cause of posterior heel pain, characterized clinically by prominent bursal bony projection ${ }^{2}$ (Figs 1 and 2). Usually, the condition is idiopathic and bilateral but there are some common provoking factors which include age, increased repetitive loading, obesity, and systemic inflammatory disease (psoriatic arthritis, spondyloarthropathy, rheumatoid arthritis, and Reiter's disease). Other infrequent factors include genetic susceptibility, malalignment in lower limbs, corticosteroids, fluoroquinolones, protease inhibitor, inflammatory disorders, connective tissue disease, vascular diseases, diabetes mellitus, hypertension, and hypercholesterolemia. It is more prevalent in a young, active population (female $>$ male) including runners and athletes. It usually affects the middle-aged people and females have a higher predisposition than males. Usually, bursal inflammation is due to impingement or mechanical irritation in the dorsiflexion between the Achilles tendon and the calcaneal tuberosity. The clinical features consist of pain at the posterior aspect of the heel which is predominantly present when the patient begins to walk after a period of rest or inactivity.

\footnotetext{
$\overline{{ }^{1} \text { Department of Orthopaedics, Abhishek Hospital and Foot Ankle }}$ Center, Vadodara, Gujarat, India

${ }^{2}$ Department of Orthopaedics, Government Medical college, Vadodara, Gujarat, India

${ }^{3}$ Department of Orthopaedics, Bodeli Dhokaliya Public Hospital, Bodeli, Gujarat, India

${ }^{4}$ Department of Orthopaedics, Surat Municipal Institute of Medical Education and Research, Surat, Gujarat, India

Corresponding Author: Malhar Dave, Department of Orthopaedics, Abhishek Hospital and Foot Ankle Center, Vadodara, Gujarat, India, Phone: +91 9825998949, e-mail: moradiyanirav@gmail.com

How to cite this article: Dave M, Dave HM, et al. Surgical Treatment of Insertional Achilles Tendinosis and Haglunds Deformity by Using Central Tendon-splitting Approach: Retrospective Case Series of 15 Cases. J Foot Ankle Surg (Asia-Pacific) 2019;6(1):18-22.

Source of support: Nil

Conflict of interest: None
}

Diagnosis is confirmed on the lateral radiographs of the ankle, which reveals a bony projection at the posterosuperior part of the calcaneal tuberosity, calcaneal bursal swelling, and increased density in the bursae. ${ }^{12}$ Magnetic resonance imaging (MRI) may be required for doubtful cases. ${ }^{13}$ Most patients will have a gastrocnemius contracture on examination.

Conservative management in the form of rest, leg elevation, cold packs, modifications of shoe wear (insoles), eccentric tendo-Achilles exercise, extracorporeal shock wave therapy, and analgesia leads to the

o The Author(s). 2019 Open Access This article is distributed under the terms of the Creative Commons Attribution 4.0 International License (https://creativecommons. org/licenses/by-nc/4.0/), which permits unrestricted use, distribution, and non-commercial reproduction in any medium, provided you give appropriate credit to the original author(s) and the source, provide a link to the Creative Commons license, and indicate if changes were made. The Creative Commons Public Domain Dedication waiver (http://creativecommons.org/publicdomain/zero/1.0/) applies to the data made available in this article, unless otherwise stated. 


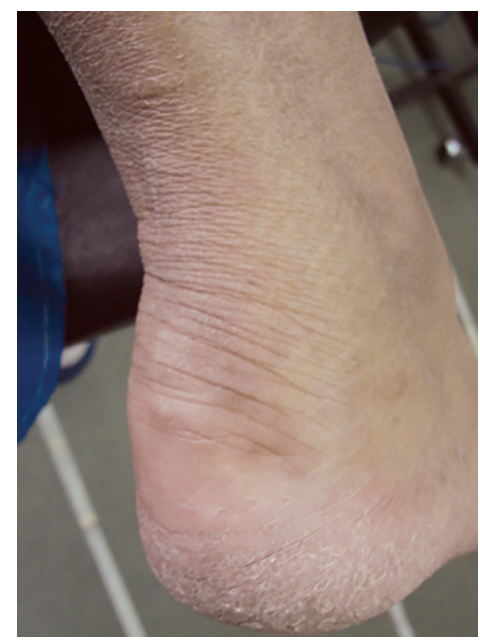

Fig. 1: Haglund's deformity clinical photograph

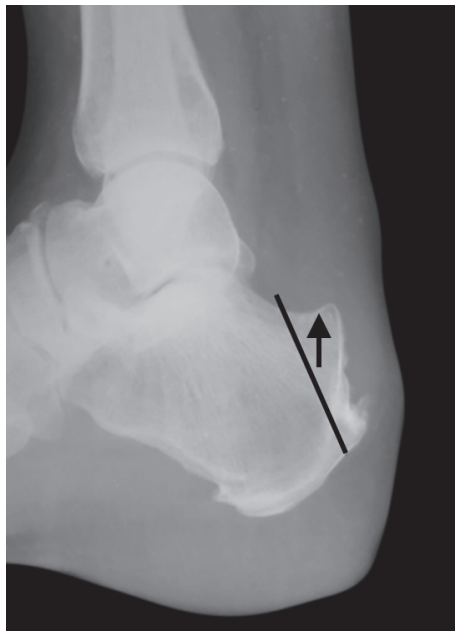

Fig. 3: Haglund's deformity lateral X-ray of hindfoot showing a prominent bursal projection of calcaneus (black arrow)

resolution of symptoms in most patients. A local steroid injection may lead to an increased risk of an acute tear, ${ }^{14}$ hence, not recommended. Casting may be necessary for pain reduction in some cases. But sometimes, it may be difficult to treat effectively by nonoperative measures alone. Surgery is a reasonable option for patients (10\%) not responding to 3-6 months of nonoperative treatment. Surgical options are posterior calcaneal osteophyte removal, excision of Haglund's deformity, limited Achilles debridement, complete debridement of the tendon insertion with bone anchor re-attachment, and isolated gastrocnemius fascia release, excision of the retrocalcaneal bursa, and calcaneus osteotomy. ${ }^{7,9}$

Several incisions have been advocated for treatment of Insertional Achilles Tendinopathy, including posterior central midline, medial, and/or lateral, J-shaped, and transverse incisions. ${ }^{15-21}$ Traditionally, surgery of the Achilles tendon is performed through longitudinal extensile incisions. The purpose of this study is to determine the effectiveness of the central tendon-splitting approach. We believe that it provides an excellent exposure to the tendon, facilitating adequate debridement of the tendon and bursa., Concerns regarding the tendon-splitting approach include wound healing, wound breakdown, and compromisation of the integrity of the tendon with slow return to full function and scar irritation about the heel counter. ${ }^{1}$

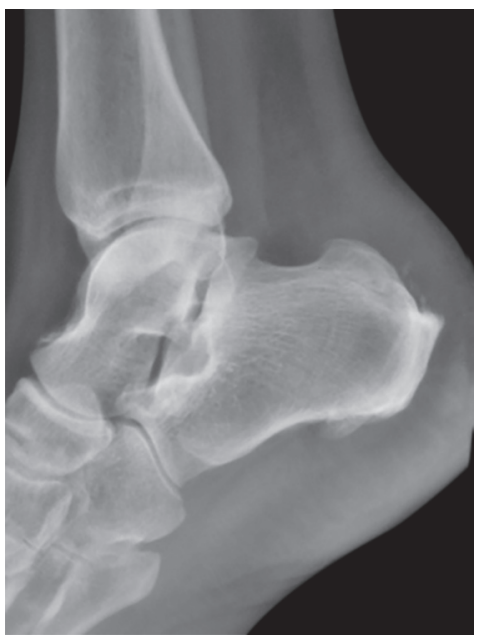

Fig. 2: Haglund's deformity-X-ray picture

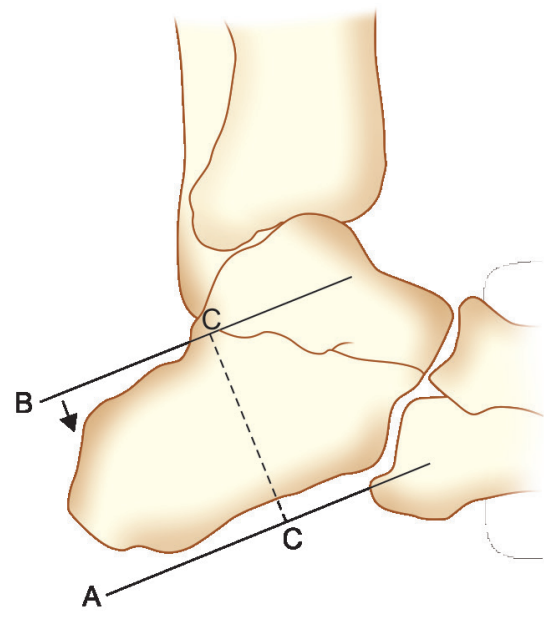

Fig. 4: Haglund's deformity - hindfoot showing area of pathologybursal projection of calcaneus (black arrow)

\section{Materials and Methods}

We studied the functional outcome of 15 patients who underwent surgical correction Haglund's deformity using the central tendonsplitting approach. Patients with a history of smoking and peripheral vascular disease were excluded from the study. Patients with a minimal follow-up of 1 year after the surgical intervention were included. Informed consent was taken from all patients to participate in the study. Patients' details like age, sex, prior history of steroid injection, and medical comorbidities like diabetes mellitus (DM) and hypertension (HT) were documented.

They were examined clinically for assessing swelling, retrocalcaneal tenderness suggestive of bursitis, range of ankle movements, and gastrocnemius tightness. Preoperative radiological evaluation was done and included a lateral radiograph of the ankle of both sides (Figs 3 and 4).

\section{Surgical Procedure}

All the procedures were performed under spinal anesthesia in the prone position with the utilization of a thigh tourniquet.

A longitudinal incision was made over the Achilles tendon, approximately $8 \mathrm{~cm}$ proximal to the Achilles tendon insertion and 


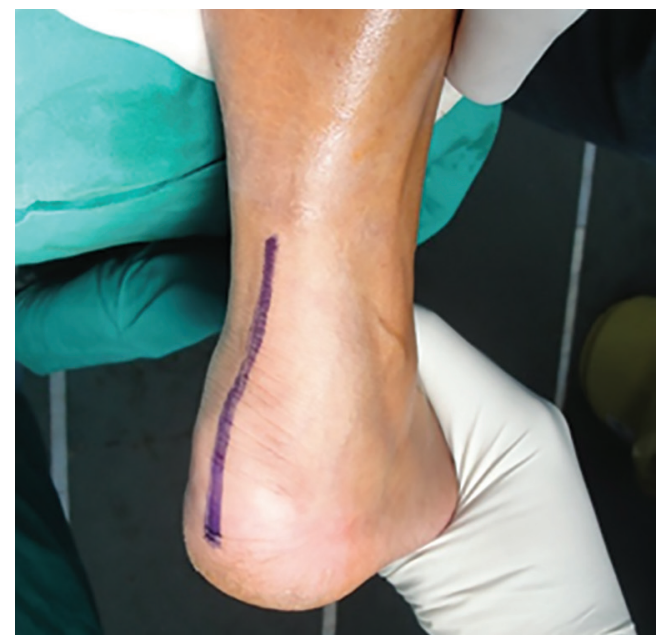

Fig. 5: Haglund's deformity—site of proposed skin incision for operative correction (marked by skin marker)

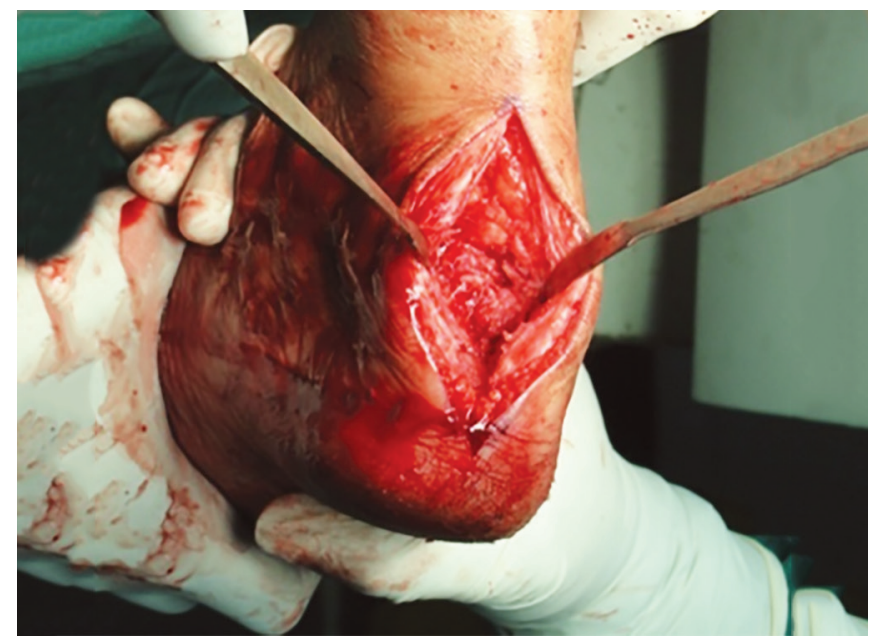

Fig. 7: Central Achilles tendon-splitting approach for operative correction of Haglund's deformity

extending distally to the glabrous skin (Fig. 5). The ankle joint was plantar flexed, and by sharp and blunt dissection, the Achilles tendon was identified. The scalpel was placed directly through the mid-line of the Achilles tendon, at the proximal end of the incision, and the scalpel is then brought straight distally (Figs 6 and 7). The retrocalcaneal bursa was resected to expose the superior aspect of the calcaneus and the posterior aspect of the subtalar joint. It was followed by excision of the superior aspect of the calcaneal tuberosity. Care was taken to excise the overlying periosteum by sharp dissection in total from the excised tuberosity to avoid new bone formation later on. Sharp edges of the tuberosity were smoothened with a rasp and rongeur. In some cases, it was necessary to resect some amount of the tendon from the calcaneum for proper exposure. All areas of fibrous degeneration and calcification then were removed from the tendon with sharp dissection. As much as $50 \%$ of tendon tissue was resected before the strength was compromised. A suture anchor size of $2.8 \mathrm{~mm}$ (Fig. 8) was used to anchor the Achilles tendon to the calcar if greater than $50 \%$ of the Achilles tendon insertion was resected. We used corkscrew-type suture anchors loaded with the number 2 Fiberwire that was inserted into the posterior tubercule of the calcaneus. One leg of the suture was woven up and down with a locking loop

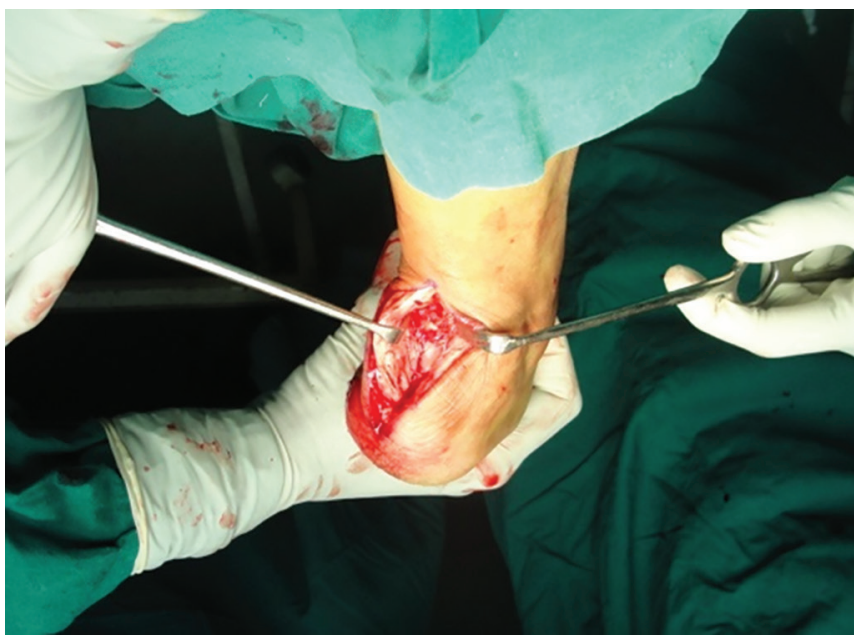

Fig. 6: Skin incision for operative correction of Haglund's deformity



Fig. 8: Use of suture anchors to anchor the Achilles tendon

technique, forming a suture column in the Achilles tendon. The second leg was passed through the distal stump and also the distal end of the tendon, in some cases. Sutures were knotted over the distal end or distal stump. The mid-line incision through the Achilles tendon was repaired with 2-0 Vicryl suture, with the knots buried on the deep aspect of the tendon. The wound then was closed using nonabsorbable sutures without drain. Tourniquet was released and spirit dressing was applied. Finally, the confirmatory image was obtained to make certain that Haglund's deformity, posterior insertional osteophytes, and all tendon calcifications have been adequately removed under an image intensifier television. The postoperative radiographs were taken in all the cases (Figs 1 and 2).

Following surgery, the patients were placed in a short leg cast with the ankle placed at $15^{\circ}$ of plantar flexion. At 2 weeks following surgery, sutures were removed and the cast was replaced with the ankle in a neutral position. The range of motion exercises was started at the end of the first month. Patients were kept nonweight-bearing for 6 weeks. Partial weight-bearing was started at 6-8 weeks. At the end of 2 months postoperatively, progressive weight-bearing as tolerated was allowed. A full return to impactloading sports activity or heavy lifting was not allowed until 6 months following surgery. 
Quantitative comparison between measures of success before and after surgery was made by the AOFAS score. The pain was measured from 0 to 40 , where 40 was no pain. The function was measured on a scale from 0 to 50 , where 50 points were normal function, and alignment was rated on a scale from 0 to 10 , where 10 was normal. ${ }^{22}$ We evaluated patients at 6 weeks, 3 months, 6 months, and 12 months after surgery.

\section{Results}

A total of 15 patients were operated. Out of them, six were women and nine were men. In nine patients, there was right heel involvement, and 6 had disease on the left side. There were no dropouts in the follow-up. The age of patients ranged from 25-60 years with a mean age of 46.9 years. The preoperative analysis showed the presence of retrocalcaneal tenderness along with pain and swelling of the calcaneum along the sides of tendon attachment suggestive of Haglund's deformity. Four patients had medical comorbidities and two patients had a history of prior steroid injection. The mean follow-up time was 26 months (range: 18-34 months). Achilles tendon calcification was evident on lateral view radiographs in $10(67 \%)$ cases.

Postoperative outcome was measured by observing the final outcome in terms of patient's satisfaction on wound dehiscence, tendon avulsion, and pain relief. The mean AOFAS score ${ }^{4}$ before the surgical intervention was 37 which increased by 33 points to a mean of 70 at the final follow-up. The mean difference in active ankle range of motion ( $\mathrm{ROM}$ ) between the operative and nonoperative extremities was $5.04^{\circ}$. There were no wound problems, painful scars, or Achilles insertion avulsions. The majority of patients in our study reported alleviation of pain and returned to activities of daily living by the end of 3 months. Of the 15 patients, 11 were very satisfied with their current foot and ankle symptoms, three were somewhat satisfied, and one was neutral (Fig. 9).

\section{Discussion}

Our study was not a randomized, controlled trial and rather reflected retrospectively collected cohorts. Patients with refractory Haglund's deformity may benefit from the calcaneal ostectomy. This study examined the success rate of a relatively less common surgery that has not been described widely in the literature. ${ }^{6-8}$ The conventional standard surgical approach is calcaneal osteotomy with a lateral

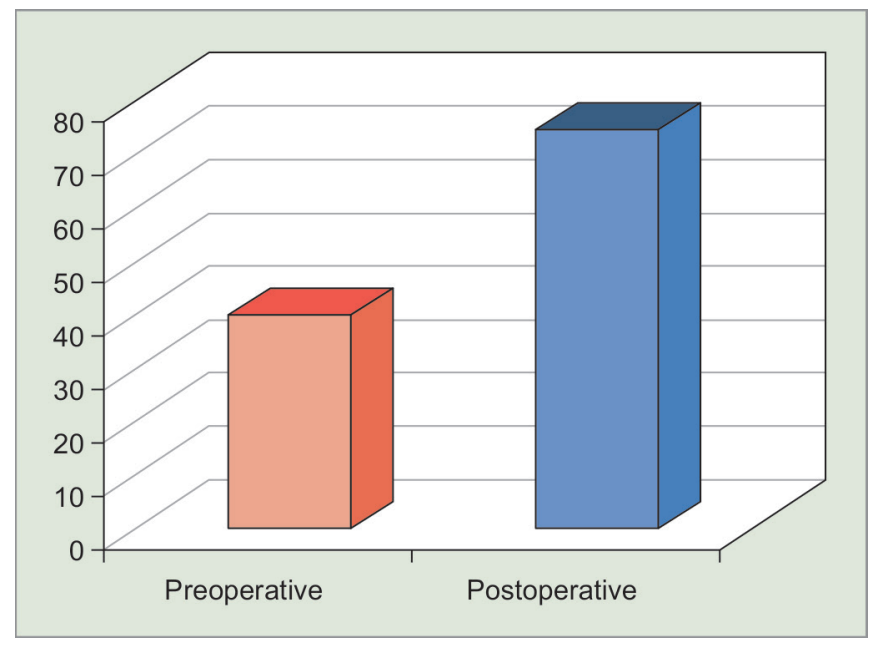

Fig. 9: Comparison of preoperative and postoperative AOFAS score longitudinal section; however, the most effective approach to this procedure has not been well defined. Other described incisions include medial, J-shaped incisions, Cincinnati incision, and a combination of both medial and lateral incisions. We, therefore, asked whether the tendon-splitting approach for a calcaneal ostectomy would result in comparable effective relief of pain, outcome scores, and complications.

Radiological parameters for Haglund's deformity usually do not carry clinical significance while planning surgical intervention. Lu et al. proved that the relation of Fowler angle and parallel pitch lines was not statistically significant between the normal heels and Haglund heels. ${ }^{23}$ Calcification of the Achilles tendon is the only radiological parameter that proves helpful to make a decision for Achilles tendon debridement while planning surgical intervention.

Our study supports surgical correction in patients with resistant cases. The mean AOFAS score was 70/100 at the final follow-up and $93 \%$ patients (14 out of 15 ) had relief of pain at 1-year followup in our series which is comparable to Samarco et al. and Sella et al. ${ }^{24,25}$ The results were, however, contradictory to Schneider et al., ${ }^{26}$ whose study had poor results with surgery.

In our study, all the patients returned to normal function by 3 months. McGarvey et al. ${ }^{29}$ and Fridrich ${ }^{3}$ used a central tendonsplitting approach that resulted in $>90 \%$ patients being able to resume routine activities by 3 months which is similar to our study. The results are surprisingly earlier compared to those reported by Natarajan et al. who reported that patients returned to normal activity by 6 months following surgery. ${ }^{27,28}$ Anderson et al. ${ }^{28}$ compared the effectiveness of tendon splitting vs the lateral group and showed that the median time to return to normal function was greater $(p=0.02)$ in the lateral incision group than in the tendon-splitting group. This suggests the superiority of the tendon-splitting approach over the lateral approach. Our key to a successful outcome is the adequate resection of the bone and probably the central tendon-splitting approach. Further, it is not possible to resect adequate bone (Sella et al. ${ }^{25}$ ) on the medial aspect while using the lateral approach. This might cause unnecessary soft tissue stretching, compromisation of vascularity, persistence of symptoms in postoperative period, and wound-healing problems.

A significant association was found between rest/avoiding sports activity during inflammation and satisfaction with surgical outcomes. This can be explained in several ways. First of all, avoiding exertion decreases mechanical damage to the inflamed tendon. In addition, it can be assumed that participants who avoided sports activity during inflammation were more compliant with medical treatment, and, therefore, have a better chance of recovery. Patients who are highly compliant would be more likely to follow the recovery protocol than patients with low compliance.

The possible disadvantage of the tendon-splitting approach is that it might result in delayed wound healing and excessive scar formation with adherence of overlying skin to tendon leading to shoe wear problems. However, these complications were not seen in our study.

This study is limited by the retrospective follow-up and possible recall bias of patients regarding their pain and functional limitations pre- and postoperatively.

\section{Suggestions}

Timely release of tourniquet, meticulous handling of soft tissues, avoiding tension on wound edges, proper wound closure, and compliance with postoperative and physiotherapy protocol are the key to our successful surgical outcomes. 


\section{Conclusion}

Our findings suggest that surgical treatment consisting of Achilles debridement through a tendon-splitting approach, excision of the calcaneal posterior insertional exostosis, and removal of the Haglund's prominence is safe and effective in relieving the pain of chronic insertion Achilles tendinopathy. Surgery produced effective relief of pain with reasonable outcomes, few complications, return to activity, and satisfaction among the patients. The radiological parameters do not carry any significance and judgment to plan for surgery should be purely on clinical grounds. A prospective study will allow a more accurate preoperative evaluation of pain and function and, therefore, a more accurate estimate of improvement.

\section{References}

1. Anderson JA, Suero E, et al. Surgery for retrocalcaneal bursitis: a tendon-splitting versus a lateral approach. Clin Orthop Relat Res 2008;466:1678-1682. DOI: 10.1007/s11999-008-0281-9.

2. Chen $\mathrm{CH}$, Huang PJ, et al. Surgical treatment for Haglund's deformity. Kaohsiung J Med Sci 2001 Aug;17(8):419-422.

3. Fridrich F. Tendon-splitting approach for the surgical treatment of Haglund's deformity and associated condition. Evaluation and results. Acta Chir Orthop Traumatol Cech 2009;76(3):212-217.

4. Rodrigues RC, Masiero D, et al. Translation, cultural adaptation and validity of the american orthopaedic foot and ankle society (AOFAS) ankle-hindfoot scale. Acta Ortop Bras. [serial on the Internet] 2008;16(2):107-111. DOI: 10.1590/S1413-78522008000200009.

5. James AN, Glenn BP, et al. Advanced Reconstruction, foot and ankle. American Academy of Orthopaedic Surgeons (AAOS), 2004.

6. Kolodziej P, Glisson RR, et al. Risk of avulsion of the Achilles tendon after partial excision for treatment of insertional tendonitis and Haglund's deformity: a biomechanical study. Foot Ankle Int 1999;20(7):433-437. DOI: 10.1177/107110079902000707.

7. Myerson MS, McGarvey W. Disorders of the Achilles tendon insertion and Achilles tendinitis. Instr Course Lect 1999;48:211-218.

8. Watson $A D$, Anderson $R B$, et al. Comparison of results of retrocalcaneal decompression for retrocalcaneal bursitis and insertional Achilles tendinosis with calcific spur. Foot Ankle Int 2000;21(8):638-642. DOI: 10.1177/107110070002100802.

9. David BT. Foot and Ankle (Orthopaedic Surgery Essentials), 2nd ed. 2012.

10. Wagner E, Gould JS, et al. Technique and Results of Achilles Tendon Detachment and Reconstruction for Insertional Achilles Tendinosis. Foot Ankle Int 2006;27(9):677-684. DOI: 10.1177/107110070602700904.

11. Astrom M, Rausing A. Chronic Achilles Tendinopathy. A Survey of Surgical and Histopathologic Findings. Clin Orthop Relat Res 1995;316:151-164. DOI: 10.1097/00003086-199507000-00021.

12. Pavlov $H$, Heneghan MA, et al. The Haglund syndrome: initial and differential diagnosis. Radiology 1982;144(1):83-88. DOI: 10.1148/ radiology.144.1.7089270.
13. Lawrence DA, Rolen MF, et al. MRI of heel pain. Am J Roentgenol 2013;200(4):845-855. DOI: 10.2214/AJR.12.8824.

14. Kleinman $M$, Gross $A E$. Achilles tendon rupture following steroid injection. Report of three cases. J Bone Surg Am 1983;65(9):1345-1347. DOI: 10.2106/00004623-198365090-00019.

15. Wagner E, Gould JS, et al. Technique and Results of Achilles Tendon Detachment and Reconstruction for Insertional Achilles Tendinosis. Foot Ankle Int 2006;27(9):677-684. DOI: 10.1177/107110070602700904.

16. Chiari Vulpiani M, Guzzini M, et al. Operative Treatment of Chronic Achilles Tendinopathy. Int Orthop 2003;27:307-310. DOI: 10.1007/ s00264-003-0472-7.

17. Yodlowski ML, Scheller Jr AD, et al. Surgical Treatment of Achilles Tendonitis by Decompression of the Retrocalcaneal Bursa and the Superior Calcaneal Tuberosity. Am J Sports Med 2002;30(3):318-321. DOI: $10.1177 / 03635465020300030301$.

18. Carmont MR, Maffulli N. Management of Insertional Achilles Tendinopathy Through a Cincinnati incision. BMC Musculoskelet Disord 2007;8:82. DOI: 10.1186/1471-2474-8-82.

19. Johnson KW, Zalavras C, et al. Surgical Management of Insertional Calcific Achilles Tendinosis with a Central Tendon Splitting Approach. Foot Ankle Int 2006;27(4):245-250. DOI: 10.1177/107110070602700404.

20. Calder JD, Saxby TS. Surgical Treatment of Insertional Achilles Tendinosis. Foot Ankle Int 2003;24(2):119-121. DOI: 10.1177/107110070302400203.

21. McGarvey WC, Palumbo RC, et al. Insertional Achilles Tendinosis: Surgical Treatment Through a Central Tendon Splitting Approach. Foot Ankle Int 2002;23(1):19-25. DOI: 10.1177/107110070202300104.

22. Kitaoka HB, Alexander IJ, et al. Clinical rating systems for the ankle-hindfoot, midfoot, hallux, and lesser toes. Foot Ankle Int 1994;15(7):349-353. DOI: 10.1177/107110079401500701.

23. Lu CC, Cheng YM, et al. Angle analysis of Haglund syndrome and its relationship with osseous variations and Achilles tendon calcification. Foot Ankle Int 2007;28(2):181-185. DOI: 10.3113/FAI.2007.0181.

24. Sammarco GJ, Taylor AL. Operative management of Haglund deformity in the non athlete: a retrospective study. Foot Ankle Int 1998;19:724-729. DOI: 10.1177/107110079801901102.

25. Sella EJ, Caminear DS, et al. Haglund syndrome. J Foot Ankle Surg 1998;37:110-114. DOI: 10.1016/S1067-2516(98)80089-6.

26. Schneider W, Niehus W, et al. Haglund syndrome: disappointing results following surgery: a clinical and radiographic analysis. Foot Ankle Int 2000;21:26-30. DOI: 10.1177/107110070002100105.

27. Natarajan S, Narayanan VL. Haglund deformity-Surgical resection by the lateral approach. Malaysian Orthop J 2015;9(1):1-3. DOI: 10.5704/ MOJ.1503.006.

28. Anderson JA, Suero E, et al. Surgery for Retrocalcaneal Bursitis: A Tendon-splitting versus a Lateral Approach. Clin Orthop Relat Res 2008;466(7):1678-1682. DOI: 10.1007/s11999-008-0281-9.

29. McGarvey WC, Palumbo RC, et al. Insertional Achilles tendinosis: surgical treatment through a central splitting approach. Foot Ankle Int 2002;23(1):19-25. DOI: 10.1177/107110070202300104. 